\title{
Proactive Motor Functional Recovery Following Immersive Virtual Reality-Based Limb Mirroring Therapy in Patients with Subacute Stroke
}

\author{
Destaw B. Mekbib ${ }^{1} \cdot$ Zhiyong Zhao ${ }^{2,3,4} \cdot$ Jianbao Wang ${ }^{5,6} \cdot$ Bin Xu $^{7,5} \cdot$ Li Zhang $^{8} \cdot$ Ruiding Cheng $^{8} \cdot$ Shan Fang $^{8}$. \\ Yuling Shao ${ }^{8}$. Wei Yang ${ }^{8}$. Jiawei Han ${ }^{9} \cdot$ Hongjie Jiang $^{9} \cdot$ Junming $\mathrm{Zhu}^{6,9} \cdot{\text { Xiangming } \mathrm{Ye}^{8} \text {. Jianmin Zhang }}^{9}$. \\ Dongrong $\mathrm{Xu}^{4}$ (1)
}

Published online: 15 July 2020

(C) The American Society for Experimental NeuroTherapeutics, Inc. 2020

\begin{abstract}
Virtual reality (VR) is considered to be a promising therapeutic technology for the rehabilitation of upper extremities (UEs) poststroke. Recently, we designed and then implemented a neuroscientifically grounded VR protocol for the rehabilitation of patients with stroke. The system provides unilateral and bilateral limb mirroring exercises in a fully immersive virtual environment that may stimulate and activate the mirror neuron system in the brain to help patients for their rehabilitation. Twelve patients with subacute stroke underwent the newly implemented VR treatment in addition to conventional rehabilitation for 8 consecutive weekdays. The treatment effect on brain reorganization and motor function was investigated using resting-state fMRI (rs-fMRI) and the Fugl-Meyer assessment for Upper Extremity (FM-UE), respectively. Fifteen healthy controls (HCs) also underwent rs-fMRI scanning one time. The study finally obtained usable data from 8 patients and $13 \mathrm{HCs}$. After the intervention, patients demonstrated significant improvement in their FM-UE scores $(p$ values $<0.042)$. Voxel-wise functional connectivity $(\mathrm{FC})$ analysis based on the rs-fMRI data found that HCs showed widespread bilateral FC patterns associated with the dominant hemispheric primary motor cortex (M1). However, the FC patterns in patients revealed intra-hemispheric association with the ipsilesional M1 seed and this association became visible in the contra-hemisphere after the intervention. Moreover, the change of FC values between the bilateral M1 was significantly correlated with the changes in FM-UE scores $(p$ values $<0.037)$. We conclude that unilateral and bilateral limb mirroring exercise in an immersive virtual environment may enhance cortical reorganization and lead to improved motor function.
\end{abstract}

Electronic supplementary material The online version of this article (https://doi.org/10.1007/s13311-020-00882-x) contains supplementary material, which is available to authorized users.

Junming Zhu

dr.zhujunming@zju.edu.cn

Dongrong $\mathrm{Xu}$

xu.dongrong@columbia.edu

1 Interdisciplinary Institute of Neuroscience and Technology, College of Biomedical Engineering and Instrument Science, Zhejiang University, Hangzhou 310000, China

2 Shanghai Key Laboratory of Magnetic Resonance, Institute of Cognitive Neuroscience, East China Normal University, Shanghai 200064, China

3 Key Laboratory for Biomedical Engineering of Ministry of Education, College of Biomedical Engineering \& Instrument Science, Zhejiang University, Hangzhou 310027, China

4 Molecular Imaging and Neuropathology Division, Department of Psychiatry, Columbia University \& New York State Psychiatric Institute, New York, NY 10032, USA
5 Key Laboratory of Biomedical Engineering of Ministry of Education, Zhejiang University, Hangzhou 310029, China

6 Department of Neurology of the Second Affiliated Hospital, Interdisciplinary Institute of Neuroscience and Technology, Zhejiang University School of Medicine, Hangzhou 310029, China

7 Interdisciplinary Institute of Neuroscience and Technology, Qiushi Academy for Advanced Studies, Second Affiliated Hospital, School of Medicine, Zhejiang University, Hangzhou 310029, China

8 Department of Rehabilitation Medicine, Zhejiang Provincial People's Hospital, People's Hospital of Hangzhou Medical College, Hangzhou 310014, China

9 The Second Affiliated Hospital, Zhejiang University School of Medicine, Zhejiang University, Hangzhou 310029, China 
Key Words Virtual reality $\cdot$ limb mirroring therapy $\cdot$ mirror neuron system $\cdot$ stroke rehabilitation $\cdot$ resting-state fMRI $\cdot$ upper extremity

\section{Introduction}

\section{Stroke}

Stroke can be defined as an interruption of blood flow within the brain that causes the death of massive brain cells [1]. Over the past decade, stroke-related mortality has rapidly declined. However, the number of neurologically impaired patients living with a substantial disability after stroke has rapidly increased [2]. Approximately $65 \%$ of stroke survivors have functional limitations on their upper extremities (UEs), which severely diminish their quality of life [3]. Evidence suggested that highly intensive and repetitive functional practices coupled with extended treatment duration are likely necessary to promote neuroplasticity in the damaged brain areas and favor the functional recovery of post-stroke UEs [4, 5]. Occupational and physical therapies may help patients improve their UE motor function [6, 7]. These treatment options, however, are time-consuming, resource-intensive, and the outcomes often depend on the ability of the medical staff who administer the treatments. Moreover, training intensity and repetition of such therapies have been evaluated as insufficient to boost neuroplasticity and associated motor recovery [8]. In contrast, virtual reality (VR)-based neurorehabilitation exercise may easily address these limitations and therefore holds a great promise in improving motor function through promoting use-dependent neuroplasticity in the damaged brain areas [9].

\section{Virtual Reality}

VR is a computer-based technology that allows users to interact with a multisensory simulated environment and receives real-time feedback on performance [8]. VR has the potential to provide highly intensive and repetitive functional tasks, and may also enhance the patient's rewarding system by adding playful elements in the paradigm. Moreover, some of the VR systems allow personalized graded programs to maintain motor capabilities by increasing the level of task difficulty [9].

\section{Basic Theory Behind VR-Based Neuro-rehabilitation}

The foremost neuroscientific theory cited in VR-based neurorehabilitation research and clinical practice is the one concerning mirror neurons (MNs). The MNs are a subset of brain cells and were initially discovered in the premotor cortex of macaque by a group of Italian scientists [10]. These scientists have initially confirmed that neurons in the premotor cortex of macaque were responsible for reaching and grasping related hand movements [11]. Furthermore, they had extended their demonstration by directly recording the neurons' activities in the premotor cortex of macaque when the macaque was performing a goal-oriented reach-to-grasp action and when the macaque was observing the same actions performed by another macaque [10]. Their demonstration revealed that hand cells (neurons responsible for hand movements) in the premotor cortex of macaque were activated and discharged both when the macaque performing motor acts as well when observing the same acts performed by another macaque [10]. Such neurons are named as "mirror neurons," which refers to neurons with mirroring properties [12].

Recent neuroimaging evidence in macaques revealed neurons with mirror properties seemed to be found in widespread motor-related brain regions, including the supplementary motor area (SMA), the premotor cortex, the primary motor cortex (M1), and the non-motor-related brain regions, including the middle frontal gyrus (MFG), the superior parietal gyrus (SPG), and the inferior parietal gyrus (IPG) [12, 13]. Human neuroimaging studies also reported that there had been neuronal activation overlaps between action execution and action observation tasks in higher-order motor-related cortical areas including the M1, SMA, premotor cortex, primary sensory cortex (S1), cerebellum, SPG, supramarginal gyrus (SMG), and inferior frontal gyrus (IFG) [13-15]. In addition, MNs also seemed to observe during imaginary motor-control acts ("brain rehearsal") [16]. For instance, using fMRI paradigm, Prochnow et al. [17] investigated the healthy subjects' brain areas controlling imaginary catching of a far distance approaching colored balls in an outdoor virtual environment. The authors confirmed that the parietal, frontal, temporal, and cerebral brain areas were activated during the target imaginary catching tasks. Hence, taking into consideration the MNs, a number of VR-based neuro-rehabilitation methods such as the rehabilitation gaming system [9], Mind Motion Pro TM (MindMaze SA, Switzerland), and YouGrabber (YouRehab Ltd., Schlieren, Switzerland) have recently been developed and implemented in the rehabilitation of stroke patients with UE deficits.

VR applications in stroke rehabilitation are ranging from custom-built virtual environment (CBVE) to commercial video gaming console (CVGC) [5]. Evidence from meta-analysis studies indicated that the use of VR-based rehabilitation protocols for UE training may be beneficial, although results were variable $[4,5,8,18]$. One possible source of variability may be due to methodological limitations of the included studies. For instance, Saposnik et al. [19] used a CVGC named Nintendo Wii gaming for the rehabilitation of patients with stroke who had impaired UEs. The authors reported that the Wii gaming console had no superior benefit in the recovery of 
UEs to a dose-matched conventional treatment. CVGC systems were originally designed for healthy people for recreation, which is limited in adjusting task difficulty. CVGC systems are in fact excessively challenging for patients with stroke [20]. Moreover, a recent meta-analysis study confirmed that the impact of CBVE on body function and activity performance had superior benefits than that of dose-matched conventional therapies, but VGC systems did not [18]. Therefore, CBVE holds a greater promise in the rehabilitation of UE motor functions. However, most of the CBVE studies did not consider the following issues: first, the majority of the studies involved in a chronic phase of stroke. Studies reported that intervention administered in the chronic phase of stroke (over 6 months from the onset of stroke) associated with poor UE recovery because neuroplasticity (the plasticity of brain functionalities) may have become less elastic at this stage [5]. In contrast, brain plasticity is high within 6 months poststroke. In turn, intervention within this time window is associated with better UE recovery $[5,15]$. Second, most studies did not use a fully immersive virtual environment, whereas the use of an immersive virtual environment for stroke rehabilitation may enhance the sense of experiencing the reality of being in another world, and may further result in a more efficient treatment on the impaired UEs [21]. Third, rehabilitation of severely impaired UE after a stroke has remained the most challenging clinical practice [22], although most of the existing VR systems do not support patients who have no sufficient power to perform training tasks related to hand opening-closing $[9,19,21]$. Fourth, despite the rise of VRbased neuro-rehabilitation studies for stroke patients with UE motor deficits, the neural mechanism for motor recovery has not yet been adequately studied.

Taking into consideration the recent advancement in VR technology and the four research gaps aforementioned, we have designed and implemented a novel type of immersive VR system, which we call it mirroring neuron VR Rehab (MNVR-Rehab) for the rehabilitation of UE motor deficit post-stroke. The MNVR-Rehab system provides game-based training tasks such as unilaterally and bilaterally grasping and releasing a virtual ball using virtual limb(s). The movements of the virtual limb were controlled by either the patient's affected UE or the reflection of the normal UE's movements (limb mirroring). In this study, we were interested in testing the impacts of VR-based limb mirroring therapy (VRLMT) on brain reorganization and UE recovery in stroke patients with moderate to severe UE impairments. We hypothesized that using our MNVR-Rehab as a VRLMT therapy in an immersive virtual environment would stimulate the MNs in the damaged brain areas, and consequently facilitate the rehabilitation of the functional use of UEs in patients with a subacute stroke. Resting-state functional magnetic resonance imaging (rs-fMRI) was used to investigate VR-induced neuroplasticity changes in the brains of patients with a stroke.

\section{Materials and Methods}

\section{Participants}

Twelve patients with subacute stroke were recruited from the inpatient service at the Department of Rehabilitation Medicine, Zhejiang Province People's Hospital (Hangzhou, China) between July 2018 and September 2019. Patients were screened and included based on the following inclusion criteria: (a) moderate to severe UE impairments due to a first-episode stroke; (b) stroke duration not exceeding 3 months; (c) adults (older than 18 years); (d) no moderate to severe visual or auditory functional impairments; (e) no moderate to severe cognitive impairments; (f) right-handed before stroke; and (g) no contraindications to MRI scanning. This study also recruited 15 righthanded HCs as a control group. This study was approved by the responsible local Ethics IRB committee at the hospital in accordance with the Helsinki Declaration, and informed written consents were obtained from all participants before their enrollment into the study.

\section{Clinical Assessment}

The FM-UE assessment was used to quantify the motor recovery status before and after the VRLMT. The FM-UE assessment is valid for quantifying the motor function as it is a widely used clinical scale in virtual reality trials and therapies [5].

\section{VR Intervention}

The MNVR-Rehab system comprises of the following elements (Fig. 1(a)): (1) A HTC Vive head-mounted display (HMD) to fully immerse the patient in the virtual environment; (2) two base stations (lighthouses) to track the patient's exact location in 3D; (3) a piece of Leap Motion to track the patient's UE movements and transfer the movements onto a virtual limb in the virtual environment; and (4) a highperformance PC with powerful graphics as the central controller, running the software system to generate the virtual environment, supervise the participant's performance, record the patient's actions, and choose various training options.

The MNVR-Rehab system provides game-based exercises of unilateral and bilateral reach-to-grasp tasks, requiring to use either the affected UE or the unaffected UE. In this study, all eligible patients underwent the MNVR-Rehab as a VRLMT training in addition to conventional treatments. According to the previous study [19], all patients have received $8 \mathrm{~h}$ of VR training and $8 \mathrm{~h}$ conventional treatment within 2 weeks (1-h VR and 1-h conventional therapy per day, 4 days/week).

Training Protocol The patient was instructed to seat on a chair comfortably, put his/her hands on a real tabletop, and wear the HMD headset to navigate the virtual objects and environment 


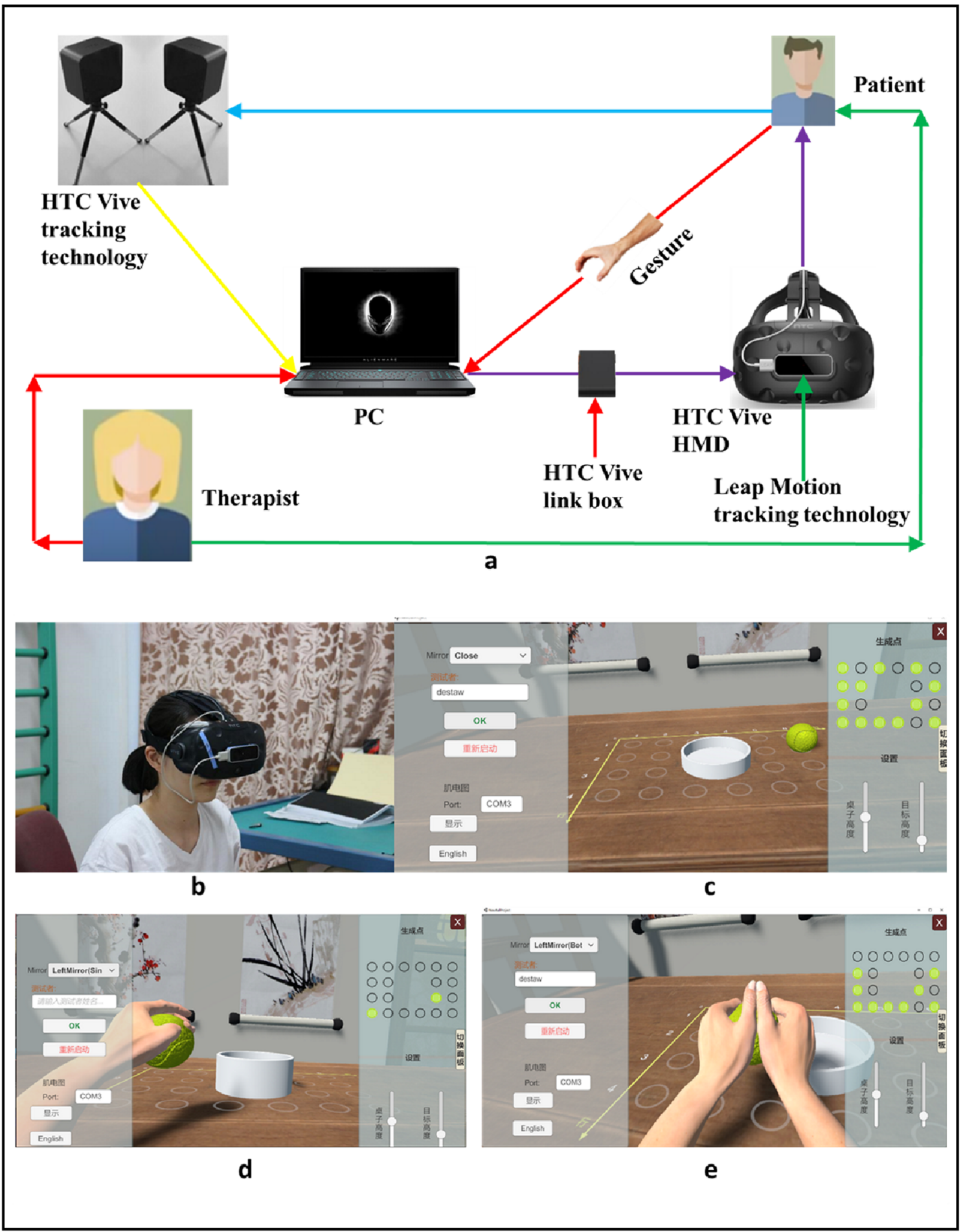

Fig. 1 Setup and scenario of the MNVR-Rehab system: (a) the arrows indicate the interaction between the system and the patient. The patient wears the HMD headset; the two infrared cameras track the 3D exact location of the patient; send the information to the PC. The leap motion controller tracks the gesture of the patient, and it graphically mimics it in the virtual environment. The PC generates the virtual environment and displays in the HMD via the HTC Vive link box and the patient immerses into and navigates the virtual environment through HMD. The therapist may assist the patient during the training and monitor his/her performance on the PC. (b) The patient wears the HMD headset and is navigating the virtual environment and objects. (c) A screenshot of a typical scene in the training environment; (d) a screenshot when the patient moves a virtual ball into a virtual basket using her left virtual limb; and (e) a screenshot when the patient moves the ball into the basket using her both virtual limbs together 
(Fig. 1(b)). In the virtual environment, he/she would see a typical scene of a training environment, including a virtual tabletop displaying a ball on it (Fig. 1(b)). Twenty equally spaced white circles were programmed to be shown in a matrix on the virtual tabletop to indicate the location of virtual balls (Fig. 1(c-e)). An aerial-view map (Fig. 1(c-e), top right) was also programmed for the therapist to define the locations where to populate the virtual balls.

Reaching and Grasping Training The therapist could choose one of the following training modes based on the patient's interest (Fig. 1(c-e), top left). (1) Unilateral limb mirroring (Fig. 1(d)): the patient was instructed to move his/her unaffected UE to guide the virtual limb, which was parallel to his/her affected UE, for the purpose of a target ball grasping and releasing training. (2) Bilateral limb mirroring (Fig. 1(e)): the patient was instructed to move his/her unaffected UE in order to coordinate the right-side and left-side virtual limbs together for the purpose of a target ball grasping and releasing training. After choosing the training mode, the therapist randomly set 20 colored balls from the aerial-view map. Then, the patient was instructed to reach, grasp, and release each ball into the basket. After completing the first VR session (20 balls), the therapist could set the next VR session and adjust the task complexity to be slightly higher, lower, or keep it as it was in the previous session based on the patient's activity performance.

\section{MRI Scanning}

A 7-T MAGNETOM scanner (Siemens Healthcare, Erlangen, Germany) was used to collect MRI data. The patients underwent MRI scanning both before and after VRLMT, whereas the HCs were scanned only once.

A multi-band gradient echo planar imaging (mb-EPI) sequence was used to collect the rs-fMRI data (1.5-mm isotropic resolution, echo time $(\mathrm{TE})=20.6 \mathrm{~ms}$, repetition time $(\mathrm{TR})=$ $2000 \mathrm{~ms}$, mb acceleration factor $=5$, GRAPA acceleration factor $=3$, flip angle $=70$ degree, $F O V=228 \mathrm{~mm} \times 225 \mathrm{~mm}$, and 90 anterior-posterior commissioner slices, number of image volumes $=160$, scanning time $=6 \mathrm{~min}$ and $24 \mathrm{~s}$ ). In total, $160 \mathrm{rs}-$ fMRI volumes were collected for each participant. T1-weighted structural images (MP2RAG: TR/TE $=2300 / 2.64 \mathrm{~ms}$, FOV = $225 \mathrm{~mm} \times 225 \mathrm{~mm}, 0.7 \mathrm{~mm}$ slice thickness, and 208 slices, scanning time $=4 \mathrm{~min}$ and $44 \mathrm{~s}$ ) were acquired for anatomical reference. Total scanning time was $11 \mathrm{~min}$ and $8 \mathrm{~s}$.

\section{Image Analysis}

The two brain hemispheres are functionally different, typically with the left hemisphere for abstract thinking (e.g., mathematics, symbols) and the right for imaginary thinking (music, art, shape recognition, etc.). Therefore, imaging analysis typically would require brains to be spatially aligned to the same sides.
However, brains with stroke are already morphologically lesioned and functionally injured, performing analyses sticking to such a requirement of aligning to the same sides will confound and even prevent the study from identifying possible alteration and improvement offered by the treatment therapies. Therefore, we first flipped the brain images of our patients in the data preprocessing step so that lesions of the brains were assigned to the same side, which has been a routine operation in similar studies [23-25]. In our study, all participants (patients and HCs) are right-handed due to left brain dominance. Three patients had right-sided lesions, while the other five had lesions in the left hemisphere. The functional and structural images of the three with right-sided lesions were flipped along the mid-sagittal plane so that the injured hemisphere corresponded to the left hemisphere for all patients. To account for symmetric confounds resulting from hemispheric difference, we also randomly selected three HCs and flipped their brain accordingly. These 6 participants' brains stayed flipped throughout the entire image analysis procedure, including functional connectivity analysis.

\section{Imaging Data Pre-Processing}

The Advanced DPARSF (http://www.restfmri.net/forum/ DPARSF) and SPM8 (http://www.fil.ion.ucl.ac.uk/spm) toolkits were used in the preprocessing, which included the following steps. The first 5 volumes of each rs-fMRI dataset were discarded to ensure steady-state longitudinal magnetization, then 155 volumes remained and were slice-time corrected. The data were realigned to the middle volume for head motion correction and co-registered to the corresponding structural image of each participant. Thus, any participants whose head motion parameters (rotation and translation) exceeded 3 degrees or $3 \mathrm{~mm}$ would be considered unusable and were therefore excluded from the subsequent preprocessing steps [26]. Next, white matter, cerebrospinal fluid, and head motion of the Friston 24-parameter model [27] were removed as nuisance variables. The noise-cleaned fMRI data were then spatially normalized to the Montreal Neurological Institute (MNI) standard space using an EPI template and resampled into a voxel size of $3 \mathrm{~mm} \times 3 \mathrm{~mm} \times 3 \mathrm{~mm}$. The data were then smoothed by convolution with an isotropic Gaussian kernel of 6-mm full width at half maximum (FWHM) to decrease spatial noise. Finally, we removed linear trends from the time courses and used temporal band-pass filtering $(0.01-0$. $1 \mathrm{~Hz}$ ) to remove the effects of low-frequency drift and highfrequency noise, such as respiratory and heart rhythms.

\section{Functional Connectivity}

Resting-state functional connectivity (FC) methods have emerged as a powerful tool to study the relationship between spatially disparate and temporally related brain regions during awake rest $[28,29]$. Two possible reasons make the FC 
attractive [30]. First, FC acquisition can be performed without complex task demands which only require the participants to remember simple instructions. Therefore, it is particularly useful for patients with moderate to severe stroke. Second, FC allows to examine regional or whole-brain neural network interaction and investigate the impacts of diseases on these neural network interaction. The most commonly used methods for analyzing the FC results are independent component analysis (ICA) and seed-based correlation analysis. ICA is a model-free and data-driven approach that analyzes the entire brain voxels that show coherent fluctuation in BOLD rs-fMRI signals, and reveal temporally coherent neural networks. ICA has been proven to be computationally higher dimensional and provides robust statistical testing. However, performing ICA is potentially more complex, which may complicate the translation of results to clinical relevance [31].

On the other hand, seed-based FC analysis is a modelbased approach that allows us to define a hypothesis-driven ROI and find the linear correlation between this ROI with the entire brain voxels [28]. This approach has proven to be technically simplified, highly reliable, easily interpretable, and effective in identifying regions that have a strong functional connection with the seed [32].

In this study, we used seed-based connectivity approach to explore possible alteration in whole-brain FC patterns following stroke and our MNVR-Rehab treatment. Previous functional MRI studies have reported that the best functional recovery of UE post-stroke is associated with the highest return of neuronal activity in the ipsilesional M1 region [25, 30, 33, 34]. Based on this knowledge, we selected M1 (dominant hemispheric M1 in HCs and ipsilesional M1 in patients) as an ROI using the SPM Anatomy toolbox. Voxel-wise FC analysis taking the M1 as a seed was performed for each participant's rs-fMRI data set. The Advanced DPARSF toolkit was used to compute the FC maps. First, the blood oxygenation level-dependent (BOLD) time series of the voxels within the ROI were averaged to obtain the reference time series for the seed area. Second, for each participant, a correlation map was obtained by computing the correlation coefficients between the reference time series and the time series of the rest of the whole-brain voxels. Finally, correlation coefficients were converted to $z$-values using Fisher's $r$-to- $z$ transformation to improve the normality [35].

\section{Statistical Analysis}

A one-sample $t$ test was employed to identify FC and thereby generate three FC maps based on the preprocessed rs-fMRI data, for the HCs, and also the patients before and after the intervention. Then, a paired $t$ test was performed to explore changes in FC results before and after the intervention. Based on the Gaussian Random Field (GRF) theory, we took results as statistically significant when $p$ value $<$ 0.001 and cluster $>50$ voxels.
Additional statistical analyses were performed using SPSS (version 22, IBM). Mean and standard deviation (mean \pm SD) were reported for baseline demographic information. A paired Wilcoxon test was used to compute improvements on FM-UE scores from pre-intervention to post-intervention. The effect size of FM-UE was calculated based on the following formula [36]:

Cohen's $d=\frac{\left|\mathrm{m}_{1}-\mathrm{m}_{2}\right|}{\sqrt{\mathrm{S}_{1}^{2}+\mathrm{S}_{2}^{2}-\left(2 \mathrm{rs}_{1} s_{2}\right)}}$

where $m_{1}, m_{2}, s_{1}$, and $s_{2}$ are the mean and SD of the FMUE values before and after the intervention, respectively, whereas $r$ is the correlation between the two conditions. The $d$ value of $0.2,0.5$, and 0.8 represents small, moderate, and large effect size, respectively [36].

According to the previous studies [30], we computed the changes in bilateral M1-M1 FC values and the changes in FMUE scores [(post-intervention - pre-intervention) / (pre-intervention)], aiming to study the relationship between the interhemispheric M1-M1 FC and motor function using Pearson's correlation ( $p$ value $<0.05$ ).

\section{Results}

Patients' demographic information and clinical outcomes are depicted in Table 1. Four patients and two HCs were excluded. We were unable to contact three of the four patients for a second MRI scan after they finished their inpatient care and the first scan. The remaining one patient complained about the MRI noise, and he wanted to withdraw from the second MRI scan. The two HCs were excluded because of their head motion greater than $3 \mathrm{~mm}$. Consequently, data from eight patients $($ age $=57.13 \pm 4.45$ years $)$ and thirteen HCs $($ age $=55 \pm$ 7.85years) entered the final statistical analysis.

\section{Clinical Outcomes}

Prior to VRLMT training, all patients had considerable deficits in their UE motor function. After the VRLMT intervention for only 2 weeks, an improvement was already observed on the FM-UE score (mean $[\mathrm{SD}]=2.5[3.8], p$ value $<0.042$; Cohen's effective size $d=0.7$, moderate effect), reflecting moderate functional recovery of the UEs.

\section{Functional Connectivity Results}

In HCs, the dominant hemispheric M1 seed was functionally connected with widespread intra- and interhemispheric brain areas (Fig. 2(a), Table 2a; and Fig. S1 in supplementary for 3D visualization). In patients, before the intervention, the $\mathrm{FC}$ patterns in relation to the ipsilesional M1 seed were 
Table 1 Demographic information and clinical outcomes

\begin{tabular}{llllllrl}
\hline Participant & Age, years & Gender & Stroke type & Lesion location & $\begin{array}{l}\text { Days since } \\
\text { stroke onset }\end{array}$ & \multicolumn{2}{c}{ FM-UE } \\
\cline { 4 - 8 } & & & & & & Pre & Post \\
\hline P1 & 59 & M & I & L, BG, PL & 28 & 5 & 7 \\
P2 & 59 & M & H & R, PCG & 85 & 6 & 6 \\
P3 & 52 & M & I & R, MFG & 44 & 8 & 9 \\
P4 & 55 & M & I & L, BG. IC & 71 & 7 & 7 \\
P5 & 56 & M & H & L, pond & 24 & 16 & 16 \\
P6 & 51 & M & I & L, BG & 9 & 5 & 6 \\
P7 & 64 & F & I & L, BG, LV & 27 & 8 & 13 \\
P8 & 61 & F & I & R, TL & 16 & 5 & 16 \\
\hline
\end{tabular}

$P=$ participant $F=$ female; $M=$ male; $I=$ ischemia; $H=$ hemorrhage; $R=$ right hemispheric lesion; $L=$ left hemispheric lesion; $P L=$ prefrontal lobule; $I C=$ internal capsule; $P C G=$ postcentral gyrus; $M F G=$ middle frontal gyrus; $B G=$ basal ganglia; $L V=$ lateral ventricle; $T L=$ temporal lobule; $F M-U E=$ the Fugle-Meyer assessment for Upper Extremity predominantly intra-hemispheric, particularly in the ipsilesional parietal, temporal, and frontal regions. Notably, the postcentral gyrus (the $\mathrm{S} 1$ region), precentral gyrus (the M1 region), SPG, MFG, superior frontal gyrus (SFG), middle occipital gyrus (MOG), IPG, SMA, STGMTG, and superior occipital gyrus (SOG) were involved (Fig. 2(b), Table 2b; and Fig. S2 in supplementary for 3D visualization). Similar FC patterns were also observed after the intervention (Fig. 2(c), Table 2c; and Fig. S3 in supplementary for 3D display). In addition, the $\mathrm{FC}$ patterns were visible in the contralesional M1, S1, and cerebellum ( $p$ value $<0.001$, cluster $\geq 50$ voxels, GRF corrected). In consistence with the previous studies [13, $15,16,37$ regarding the brain areas showing $\mathrm{MN}$ properties, our findings also showed the M1, S1, cerebellum, SPG, SMA, and MFG regions were highlighted after the VR intervention.

\section{Changes in FC Map from Pre-intervention to Post- Intervention}

The paired $t$ statistics revealed that no significant FC changes were observed between the FC before and after the treatment at a $p$ value $<0.001$, cluster $\geq 50$ voxels, GRF corrected (Fig. 3(a)). However, when we adopted $p$ value $<0.01$, cluster $\geq 50$ voxels, GRF corrected, the paired $t$ statistics revealed significantly increased FC patterns in the bilateral M1, S1, and ipsilesional SPG, and cerebellum regions (Fig. 3(b); Table 3a) and decreased in the ipsilesional MFG and STG regions (Fig. 3(b); Table 3b; and Fig. S4 in supplementary for $3 \mathrm{D}$ visualization).

\section{VRLMT-Induced Change in FC Values and FM-UE Score}

Pearson's correlation showed that the change of the bilateral M1-M1 FC value was positively and significantly correlated with the change of FM-UE score (Fig. $4, R^{2}$ statistics $=0.729$, $p$ value $<0.037$ ), which infers a positive sign of the recovery of UE motor function due to the increased connectivity in relation to interhemispheric M1.

\section{Discussion}

In this study, we demonstrated the effect of a 2-week VRLMT training plus a conventional treatment on cortical reorganization and associated motor function in patients with subacute stroke who had moderately to severely impaired UE functions. The patients and HCs had undergone the rs-fMRI scanning twice (before and after VRLMT) and once, respectively. Overall, patients exhibited significant improvements in their affected UE motor function. In HCs, widespread bilateral brain areas were connected with the dominant hemispheric M1 seed. In the patients, before VRLMT, the FC pattern associated with the ipsilesional M1 seed was predominantly intra-hemispheric. Compared with the pre-intervention FC results, the interhemispheric FC pattern after VRLMT became more explicit in the patients between the ipsilesional M1 seed and contralesional brain regions related to motor control and sensation information processing. Moreover, a significant positive correlation was observed in the patient group between the changes in interhemispheric M1-M1 FC values and FMUE scores.

\section{VRLMT-Induced Motor Function Recovery}

One novelty aspect of this study was that we investigated the impact of unilateral and bilateral limb mirroring training in an immersive virtual environment on motor functional recovery in post-stroke patients with moderately to severely impaired UEs. The motor outcomes showed that patients got small, but statistically significant improvements on their UE motor 
a

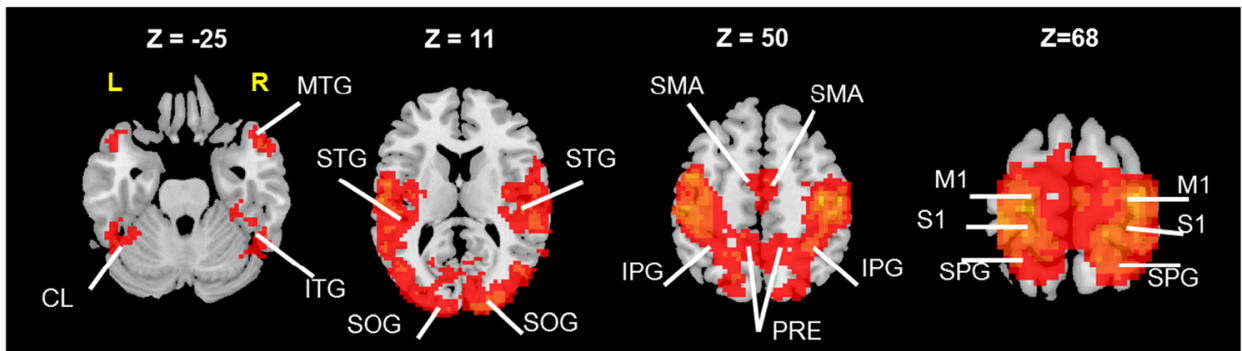

b
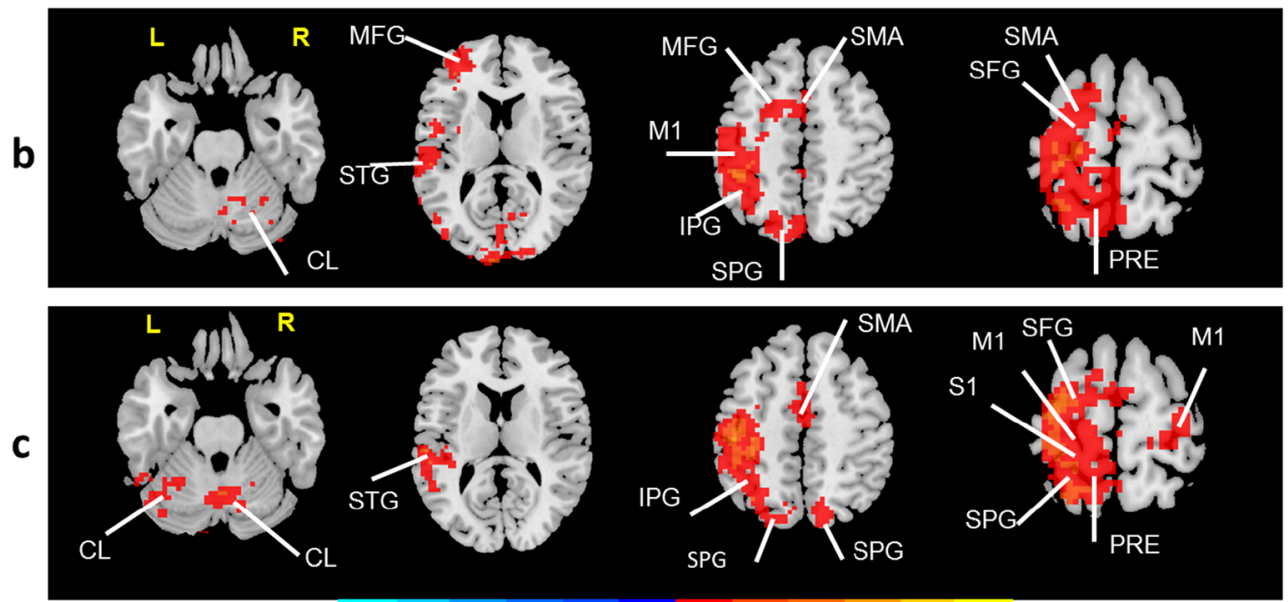

$-5 \quad-3$

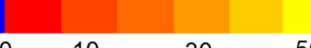

Fig. 2 FC maps based on the one-sample $t$ statistics at 4 representative transversal planes where MNI coordinates $Z=-25,11,50,68$, from the left to the right column. (a) The HCs' FC maps associated with the dominant hemispheric M1 seed and the whole brain. (b, c) The FC maps of patients before and after intervention associated with the ipsilesional M1 seed and whole brain, respectively ( $p$ value $<0.001$, cluster $\geq 50$ voxels, GRF corrected). The cold color spectrum indicates significantly decreased FC maps in relation to M1 seed and the warm color spectrum

indicates significantly increased FC maps in relation to M1 seed. Note: $\mathrm{CL}=$ cerebellum; $\mathrm{ITG}=$ inferior temporal gyrus; $\mathrm{IPG}=$ inferior parietal gyrus; $\mathrm{PRE}=$ precuneus; $\mathrm{L}=$ left $\mathrm{R}=$ right $\mathrm{MTG}=$ middle temporal gyrus; $\mathrm{STG}=$ superior temporal gyrus; $\mathrm{SPG}$ : superior parietal gyrus; $\mathrm{S} 1$ : primary sensory cortex; M1: primary motor cortex; MFG: middle frontal gyrus; SFG: superior frontal gyrus; SMA: supplementary motor area; SOG: superior occipital gyrus

function just in 2 weeks as assessed by the FM-UE clinical scale. This improvement indicated that practicing using a

goal-specific unilateral and bilateral limb mirroring exercise in an immersive virtual environment may facilitate the

Table 2 Clusters showing significantly increased FC in relation to M1 seed ( $p$ value $<0.001$, cluster $\geq 50$ voxels, GRF corrected)

Cluster no. Cluster size $\left(\mathrm{mm}^{3}\right)$ Peak MNI coordinate Peak AAL brain atlas

$$
\begin{array}{llll}
X & Y \quad Z & &
\end{array}
$$

a. HCs

$$
1 \quad 14,327
$$$$
\begin{array}{llll}
-24 & -21 & 72 & 24.17
\end{array}
$$

L: M1, S1, STG, MTG, SPG, SMA, IPG, SFG, ITG,

CL; R: M1, S1, STG, SPG, MTG, SMA, PRE, ITG, IPG, SFG

\begin{tabular}{|c|c|c|c|c|c|c|}
\hline 1 & 5916 & -33 & -36 & 60 & 43.71 & L: M1, S1, SPG, PRE, IPG, STG, SFG, SMA, CL, MTG; R: PRE, CL, SOG, MOG \\
\hline 2 & 357 & -36 & 42 & 12 & 15.85 & L: MFG, IFG \\
\hline 3 & 122 & -60 & -57 & 15 & 10.41 & L: MTG \\
\hline \multicolumn{7}{|c|}{ After intervention } \\
\hline 1 & 3421 & -33 & -24 & 63 & 27.67 & L: M1, S1, SPG, IPG, PRE, SMA, STG, MTG, SFG, SOG; R: PRE \\
\hline 2 & 666 & 27 & -54 & -30 & 14.13 & L: CL; R: CL \\
\hline 3 & 107 & 30 & -21 & 69 & 7.07 & $\mathrm{R}: \mathrm{M} 1, \mathrm{~S} 1$ \\
\hline
\end{tabular}

b. Before intervention

$A A L$ : automated anatomical labeling; $C L$ : cerebellum; $L$ : left hemispheric; $R$ : right hemispheric 

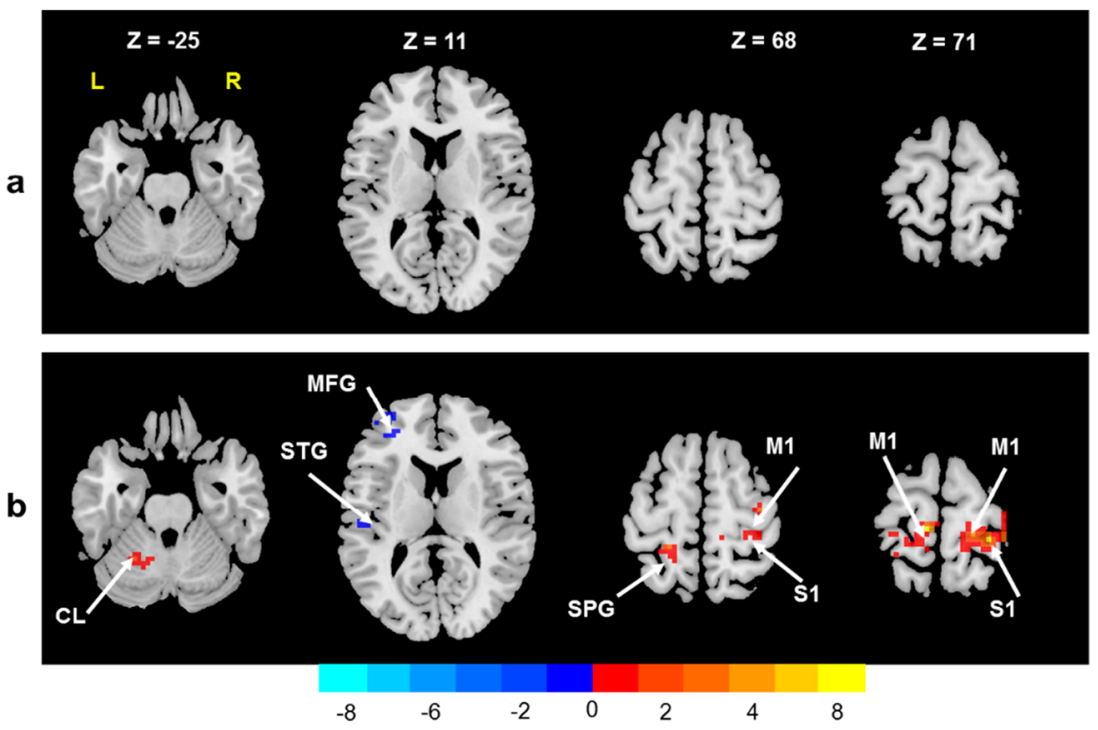

Fig. 3 Post-treatment versus pre-treatment paired $t$ statistics of the FC maps obtained from 4 representative transversal planes at MNI coordinates $Z=-25,11,68$, and 71. (a) The FC maps GRF-corrected at $p$ value greater than 0.001 and cluster $\geq 50$ voxels. (b) The FC maps GRFcorrected at $p$ value less than 0.01 and cluster $\geq 50$ voxels. The cold color spectrum indicates significant negative changes of FC maps in relation to

recovery of impaired UEs. Functional improvements of the UEs in response to VR-based limb mirroring training were investigated in previous neuro-rehabilitation studies [38, 39]. A study reported by [38] demonstrated the effect of VR-based reflection therapy on UE motor function as compared to dosematched conventional therapy that after 4 weeks of treatment, the VR group got improved as assessed by the FM-UE scores better than did the control group. Our findings differed from the findings reported by [39], which reported that using an immersive VR-based unilateral limb mirroring therapy system statistically had no significant impacts on the recovery of UE motor function in patients with chronic stroke. Our findings also have been consistent with those therapies using a physical mirror [40-42], as all the three studies investigated the impact of mirror visual feedback in the recovery of UE motor function
M1 seed between the before and after the intervention, and the warm color spectrum indicates significant positive changes of FC maps in relation to M1 seed between the before and after the intervention. Note: $\mathrm{CL}=$ cerebellum; $\mathrm{L}=$ left, $\mathrm{R}=$ right; $\mathrm{STG}=$ superior temporal gyrus; $\mathrm{SPG}=$ superior parietal gyrus; $\mathrm{S} 1$ = primary sensory cortex; $\mathrm{M} 1$ = primary motor cortex; $\mathrm{MFG}=$ middle frontal gyrus

in patients with chronic stroke, and all the three reported statistically significant improvements on the FM-UE scale.

Apparently, growing pieces of evidence are showing that VR-based, as well as mirror-based, limb mirroring therapies have significant benefits in improving the functional recovery of UEs post-stroke. Our findings also confirmed that VRLMT has significant benefits in the recovery of UE motor function in patients with subacute stroke.

\section{VRLMT-Induced Brain Reorganization}

A second novelty aspect of this study was that we investigated the neural mechanisms of VRLMT-induced UE motor functional improvements. Compared with the $\mathrm{HCs}$, the FC results
Table 3 Post-treatment versus pre-treatment comparison of FC in relation to $\mathrm{M} 1$ seed and the whole brain $(p$ value $<0.01$, cluster $\geq 50$ voxels, GRF corrected)

\begin{tabular}{llllll}
\hline Cluster & $\begin{array}{l}\text { Cluster size } \\
\left(\mathrm{mm}^{3}\right)\end{array}$ & \multicolumn{2}{l}{ Peak MNI coordinate } & Peak $t$-value \\
\cline { 3 - 5 } & & $X$ & $Y$ & $Z$
\end{tabular}

a. FC after intervention versus before intervention: positive results

\begin{tabular}{ccccccc}
1 & 63 & -22 & -56 & -25 & 3.92 & L: Cerebellum \\
2 & 165 & 27 & -33 & 72 & 7.20 & R: M1, S1 \\
3 & 88 & -9 & -27 & 72 & 8.14 & L: M1, SPG \\
b. FC after intervention versus before intervention: negative results & & L: MFG \\
2 & 132 & -29 & 45 & 11 & -4.80 & L: STG \\
3 & 205 & -53 & -19 & 11 & -3.94 & \\
\hline
\end{tabular}

$L$ : left hemispheric; $R$ : right hemispheric; $A A L$ : automated anatomical labeling 


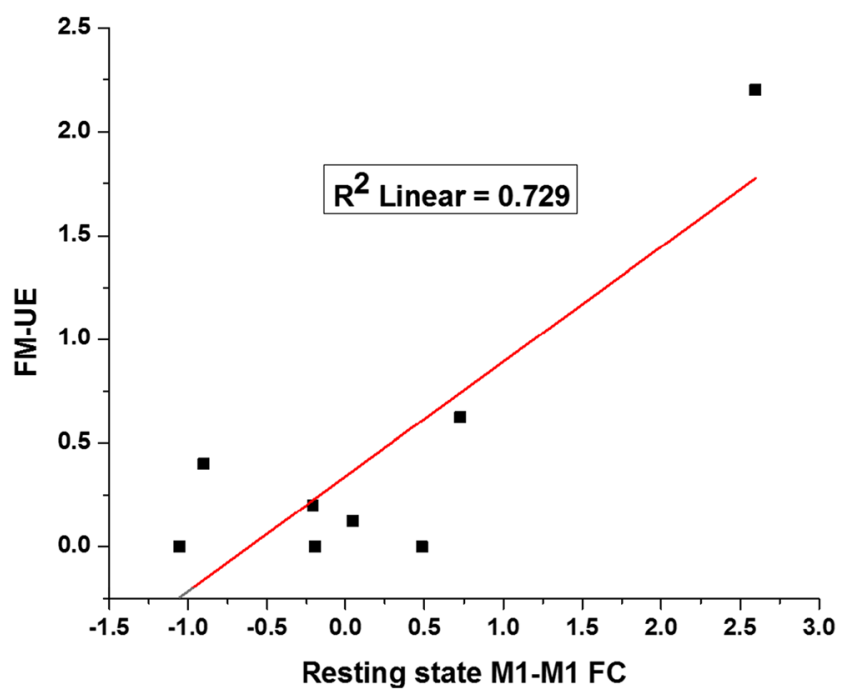

Fig. 4 Significant positive correlation between the changes in bilateral M1 FC values and the FM-UE score

in the patients showed a significant decrement in the bilateral M1 regions. M1 is a key motor area and has a function of controlling proper movements of the UEs [2]. Past studies reported that stroke-induced lesions in motor-related regions could disrupt the bilateral M1 connectivity, leading to a motor functional deficit of the UEs [30, 34, 43, 44]. During the recovery process, the interhemispheric connectivity between the $\mathrm{M} 1$ regions can be established [30, 34, 43]. Our findings based on our MNVR-rehab system are along with this that after 2 weeks of the VRLMT training the bilateral M1 connectivity was enhanced. Similar results were also documented previously in the literature [45], which reported that patients with stroke lost the resting-state FC between the bilateral M1 regions, whereas the connectivity got significantly enhanced after a motor imaginary treatment for 4 weeks. In accordance with our findings, Fan et al. [30] reported that the bilateral M1 connectivity was lost before the patients received roboticassisted training. Then the lost connectivity was reestablished after receiving a 4 -week training session.

\section{Relationship Between VRLMT-Induced Bilateral M1 Connectivity and Motor Function}

A growing body of evidence showed that interhemispheric functional connectivity between bilateral sensorimotor cortices may predict the recovery of motor function $[1,46]$. Grefkes et al. [2] reported that impaired motor performance induced by stroke was associated with a loss of interhemispheric sensorimotor connectivity, whereas the recovery of the impaired motor function was associated with improved bilateral sensorimotor connectivity. Similar findings were reported also by Fan et al. [30], which investigated the effect of a 4-week robotic-assisted treatment on brain reorganization and functional performance recovery in patients with chronic stroke. They found a statistically significant correlation between the bilateral M1 connectivity and the UE motor performance. In line with this report, our unique MNVR-rehab system also promotes neuroplasticity in the injured and distant brain areas, which may cause a significant improvement in UE motor function. Moreover, the bilateral M1-M1 FC scores were significantly correlated with the UE motor functional recovery. Our findings indicated that there were behavioral relevant plastic changes in the ipsilesional sensorimotor network. In accordance with our findings, Zhang et al. [34] reported that patients with stroke significantly improved functional use of the affected UE after 4 weeks of motor imaginary intervention, and the gained improvement was significantly coupled with the bilateral M1 functional connectivity.

Highly repetitive and intensive functional tasks are believed to be a key factor for promoting the induction of usedependent neuroplasticity, which may be associated with optimal motor recovery $[5,8,9]$. In addition, a user-controllable, motivated, and joyful rehabilitation setting is likely necessary for motor development and prolonged active training [9]. Based on these ideas, our system has been developed to provide completely immersive, joyful, game-based repetitive limb mirroring exercises. The system also provides progressive task complexity as well as visual and auditory feedback regarding successful movements, which may further increase patients' exposure to more intensive and repetitive functional practices. We believe that these unique features provided by our MNVR-Rehab system are likely promoting and activating the mirror neurons, and their connectivity in the damaged brain areas could consequently play a crucial role in helping patients for their UE functional rehabilitation [9, 15, 47]. Moreover, the options of bilateral limb mirroring training available in our MNVR-Rehab system may facilitate the recovery of the UE function. Previous studies have reported that performing repetitive functional tasks using both affected and unaffected UEs simultaneously are useful in improving the affected UE motor function through adapting the temporal and spatial characteristics of the normal UE [48].

\section{Limitation}

This study has some limitations. First, the number of participants was relatively small [34]. Because of our restrictive inclusion criteria, it was challenging to recruit a large number of participants. While the effect size of FM-UE was moderate $(d=0.7)$, reflecting the study was moderately powered. Future studies are encouraged to replicate the current study with a large number of participants that permit different potential moderators to enhance the generalizability of the current results. Second, all patients received conventional therapies alongside with our VRLMT. Hence, this study lacked a control group consisting of post-stroke patients, who received only $\mathrm{CT}$, to control for the bias that could have been induced 
by the CT and thus to identify and verify the effect on rehabilitation purely due to our VR therapy with more confidence.

The strength of our trial is that our MNVR-Rehab system has a fundamental and valid neuroscience hypothesis, which aims to activate the mirror neurons to promote neuroplasticity in the damaged brain regions. Our future studies will recruit a large number of participants with less inter-subject variations and more appropriately designed controls, and follow up them for a longer period for more detailed evaluations.

\section{Conclusion}

This study has demonstrated the effect of immersive VRbased limb mirroring therapy plus routine treatment on cortical reorganization and motor function rehabilitation in subacute stroke patients. Our findings have revealed that unilateral and bilateral limb mirroring exercises in an immersive virtual environment may stimulate MNs in the damaged brain areas and may facilitate functional recovery of the affected upper extremities post-stroke. This clinical outcome implies that therapeutic exercises based on unilateral and bilateral limb mirroring provided by our MNVR-Rehab system may be useful interventions for rehabilitation of subacute stroke patients with UE functional impairments.

Acknowledgments This work was supported in part by a grant sponsored by the China National Key R\&D Program (No. 2017YFC1308500/ 2017YFC1308502) and in part by the China National Natural Science Foundation (Grant numbers: 71971066,81471734, and 31627802). This work was also supported partly by an international collaboration grant sponsored by the China National Ministry of Science and Technology (No. 4-9/2018), and a grant sponsored by the China National Ministry of Education (No. 18YJA630019). The authors would like to acknowledge the help and assistance from Ye Li's team in support of developing the VR system in terms of choosing and connecting the peripheral VR device.

\section{References}

1. Sampaio-Baptista C, Sanders Z-B, Johansen-Berg H. Structural Plasticity in Adulthood with Motor Learning and Stroke Rehabilitation. Annu Rev Neurosci 2018;41(1):25-40.

2. Grefkes C, Fink GR. Connectivity-based approaches in stroke and recovery of function. Lancet Neurol 2014;13(2):206-16.

3. Abo M, Kakuda W, Momosaki R, Harashima H, Kojima M, Watanabe S, et al. Randomized, multicenter, comparative study of NEURO versus CIMT in poststroke patients with upper limb hemiparesis: The NEURO-VERIFY Study. Int J Stroke 2014;9(5):607-12.

4. Laver K, Lange B, George S, Deutsch J, Saposnik G, Crotty M. Virtual reality for stroke rehabilitation. Cochrane Database Syst Rev 2017;1-180.

5. Mekbib DB, Han J, Zhang L, Fang S, Jiang H, Zhu J, et al. Virtual reality therapy for upper limb rehabilitation in patients with stroke: a meta-analysis of randomized clinical trials. Brain Inj [Internet].
2020;34(4):456-65. Available from: https://doi.org/10.1080/ 02699052.2020 .1725126

6. Steultjens EMJ, Dekker J, Bouter LM, Van de Nes JCM, Cup EHC, Van den Ende CHM. Occupational therapy for stroke patients: A systematic review. Stroke. 2003;34(3):676-86.

7. Ackerley SJ, Byblow WD, Barber PA, MacDonald H, McIntyreRobinson A, Stinear CM. Primed physical therapy enhances recovery of upper limb function in chronic stroke patients. Neurorehabil Neural Repair 2016;30:319-48.

8. Saposnik G, Levin M. Virtual reality in stroke rehabilitation: A meta-analysis and implications for clinicians. Stroke. 2011;42(5): $1380-6$.

9. Da Silva Cameiro M, Bermúdez I Badia S, Duarte E, Verschure PFMJ. Virtual reality based rehabilitation speeds up functional recovery of the upper extremities after stroke: A randomized controlled pilot study in the acute phase of stroke using the Rehabilitation Gaming System. Restor Neurol Neurosci 2011;29(5):287-98.

10. Rizzolatti G, Fadiga L, Gallese V, Fogassi L. Premotor cortex and the recognition of motor actions. Cogn Brain Res 1996;3(2):13141.

11. Rizzolatti G, Camarda R, Fogassi L, Gentilucci M, Luppino G, Matelli M. Functional organization of inferior area 6 in the macaque monkey. Exp Brain Res 1988;71(3):491-507.

12. Rizzolatti G, Sinigaglia $C$. The mirror mechanism: A basic principle of brain function. Nat Rev Neurosci 2016;17(12):757-65.

13. Filimon F, Rieth CA, Sereno MI, Cottrell GW. Observed, executed, and imagined action representations can be decoded from ventral and dorsal areas. Cereb Cortex 2015;25(9):3144-58.

14. Modroño C, Navarrete G, Rodríguez-Hernández AF, GonzálezMora JL. Activation of the human mirror neuron system during the observation of the manipulation of virtual tools in the absence of a visible effector limb. Neurosci Lett 2013;555(March):220-4.

15. Brunner IC, Skouen JS, Ersland L, Grüner R. Plasticity and response to action observation: A longitudinal fMRI study of potential mirror neurons in patients with subacute stroke. Neurorehabil Neural Repair 2014;28(9):874-84.

16. Iacoboni M, Dapretto $M$. The mirror neuron system and the consequences of its dysfunction. Vol. 7, Nat Rev Neurosci. 2006. p. 942 51.

17. Prochnow D, Bermúdez i Badia S, Schmidt J, Duff A, Brunheim S, Kleiser R, et al. A functional magnetic resonance imaging study of visuomotor processing in a virtual reality-based paradigm: Rehabilitation Gaming System. Eur J Neurosci 2013;37(9):1441-7.

18. Maier M, Rubio Ballester B, Duff A, Duarte Oller E, Verschure PFMJ. Effect of Specific Over Nonspecific VR-Based Rehabilitation on Poststroke Motor Recovery: A Systematic Meta-analysis. Neurorehabil Neural Repair 2019;33:112-29.

19. Saposnik G, Cohen LG, Mamdani M, Pooyania S, Ploughman M, Cheung D, et al. Efficacy and safety of non-immersive virtual reality exercising in stroke rehabilitation (EVREST): a randomised, multicentre, single-blind, controlled trial. Lancet Neurol 2016;15(10):1019-27.

20. Teo W-P, Muthalib M, Yamin S, Hendy AM, Bramstedt K, Kotsopoulos E, et al. Does a Combination of Virtual Reality, Neuromodulation and Neuroimaging Provide a Comprehensive Platform for Neurorehabilitation? - A Narrative Review of the Literature. Front Hum Neurosci 2016;10:1-15.

21. Brunner I, Skouen JS, Hofstad H, Aßmus J, Becker F, Sanders AM, et al. Virtual reality training for upper extremity in subacute stroke (VIRTUES): A multicenter RCT. Neurology. 2017;89(24):241321.

22. Colomer C, Noé E, Llorens R. Mirror therapy in chronic stroke survivors with severely impaired upper limb function: A randomized controlled trial. Eur J Phys Rehabil Med 2016;52(3):271-8. 
23. Volz LJ, Sarfeld AS, Diekhoff S, Rehme AK, Pool EM, Eickhoff $\mathrm{SB}$, et al. Motor cortex excitability and connectivity in chronic stroke: a multimodal model of functional reorganization. Brain Struct Funct 2015;220(2):1093-107.

24. Bannister LC, Crewther SG, Gavrilescu M, Carey LM. Improvement in touch sensation after stroke is associated with resting functional connectivity changes. Front Neurol. 2015;6(JUL):1-15.

25. Wang LE, Tittgemeyer M, Imperati D, Diekhoff S, Ameli M, Fink GR, et al. Degeneration of corpus callosum and recovery of motor function after stroke: A multimodal magnetic resonance imaging study. Hum Brain Mapp 2012;33(12):2941-56.

26. Liu Y, Liang M, Zhou Y, He Y, Hao Y, Song M, et al. Disrupted small-world networks in schizophrenia. Brain. 2008;131(4):94561.

27. Friston KJ, Williams S, Howard R, Frackowiak RSJ, Turner R. Movement-related effects in fMRI time-series. Magn Reson Med 1996;35(3):346-55.

28. Biswal B, Yetkin FZ, Haughton VM, Hyde JS. Functional connectivity in the motor cortex resting human brain using echo-planar MRI. Magn Reson Med 1995;34:37-541.

29. Zang YF, Yong H, Chao-Zhe Z, Qing-Jiu C, Man-Qiu S, Meng L, et al. Altered baseline brain activity in children with ADHD revealed by resting-state functional MRI. Brain and Development 2007;29(2):83-91.

30. Fan YT, Wu CY, Liu HL, Lin KC, Wai YY, Chen YL. Neuroplastic changes in resting-state functional connectivity after stroke rehabilitation. Front Hum Neurosci. 2015;9(OCT):1-12.

31. van den Heuvel MP, Hulshoff Pol HE. Exploring the brain network: A review on resting-state fMRI functional connectivity. Eur Neuropsychopharmacol [Internet] 2010;20(8):519-34. Available from: https://doi.org/10.1016/j.euroneuro.2010.03.008

32. Dipasquale O, Milano P, Onlus G. Network functional connectivity and whole-brain functional connectomics to investigate cognitive decline in neurodegenerative conditions. Funct Neurol 2016;31(4): 191-203.

33. Zhao Z, Wang X, Fan M, Y in D, Sun L, Jia J, et al. Altered effective connectivity of the primary motor cortex in stroke: A resting-state fMRI study with granger causality analysis. PLoS One 2016;11(11):1-16.

34. Zhang Y, Liu H, Wang L, Yang J, Yan R, Zhang J. Relationship between functional connectivity and motor function assessment in stroke patients with hemiplegia : a resting-state functional MRI study. Neuroradiology. 2016;58:503-11.

35. Lowe MJ, Mock BJ, Sorenson JA. Functional connectivity in single and multislice echoplanar imaging Using Resting-State Fluctuations2. Neuroimage. 1998;7(7):119-32.

36. Yin CW, Sien NY, Ying LA, Chung SFCM, Tan May Leng D. Virtual reality for upper extremity rehabilitation in early stroke: A pilot randomized controlled trial. Clin Rehabil 2014;28(11):110714.
37. Carvalho D, Teixeira S, Lucas M, Yuan TF, Chaves F, Peressutti C, et al. The mirror neuron system in post-stroke rehabilitation. Int Arch Med 2013;6(1):1-7.

38. In TS, Jung KS, Lee SW, Song CH. Virtual reality reflection therapy improves motor recovery and motor function in the upper extremities of people with chronic stroke. J Phys Ther Sci 2012;24(4): 339-43.

39. Weber LM, Nilsen DM, Gillen G, Yoon J, Stein J. Immersive virtual reality mirror therapy for upper limb recovery following stroke. Am J Phys Med Rehabil 2019;98:1.

40. Michielsen ME, Selles RW, Van Der Geest JN, Eckhardt M, Yavuzer G, Stam HJ, et al. Motor recovery and cortical reorganization after mirror therapy in chronic stroke patients: A phase II randomized controlled trial. Neurorehabil Neural Repair 2011;25(3):223-33.

41. Samuelkamaleshkumar S, Reethajanetsureka S, Pauljebaraj P, Benshamir B, Padankatti SM, David JA. Mirror therapy enhances motor performance in the paretic upper limb after stroke: A pilot randomized controlled trial. Arch Phys Med Rehabil 2014;95(11): 2000-5.

42. Pervane Vural S, Nakipoglu Yuzer GF, Sezgin Ozcan D, Demir Ozbudak S, Ozgirgin N. Effects of Mirror Therapy in Stroke Patients with Complex Regional Pain Syndrome Type 1: A Randomized Controlled Study. Arch Phys Med Rehabil [Internet] 2016;97(4):575-81. Available from: https://doi.org/10.1016/j. apmr.2015.12.008

43. Golestani AM, Tymchuk S, Demchuk A, Goodyear BG. Longitudinal evaluation of resting-state fMRI after acute stroke with hemiparesis. Neurorehabil Neural Repair 2013;27(2):153-63.

44. Carter AR, Astafiev S V., Lang CE, Connor LT, Rengachary J, Strube MJ, et al. Resting interhemispheric functional magnetic resonance imaging connectivity predicts performance after stroke. Ann Neurol 2010;67:365-75.

45. Hatem SM, Saussez G, della Faille M, Prist V, Zhang X, Dispa D, et al. Rehabilitation of Motor Function after Stroke: A Multiple Systematic Review Focused on Techniques to Stimulate Upper Extremity Recovery. Front Hum Neurosci. 2016;10:1-22.

46. Park CH, Chang WH, Ohn SH, Kim ST, Bang OY, Pascual-Leone $\mathrm{A}$, et al. Longitudinal changes of resting-state functional connectivity during motor recovery after stroke. Stroke. 2011;42(5):1357-62.

47. Nojima I, Mima T, Koganemaru S, Thabit MN, Fukuyama H, Kawamata T. Human motor plasticity induced by mirror visual feedback. J Neurosci 2012;32(4):1293-300.

48. Choo PL, Gallagher HL, Morris J, Pomeroy VM, van Wijck F. Correlations between arm motor behavior and brain function following bilateral arm training after stroke: A systematic review. Brain Behav 2015;5(12):1-25.

Publisher's Note Springer Nature remains neutral with regard to jurisdictional claims in published maps and institutional affiliations. 